\title{
DIAMETERS IN TYPICAL CONVEX BODIES
}

\author{
IMRE BÁRÁNY AND TUDOR ZAMFIRESCU
}

1. Introduction. The most usual diameters in the world are those of a sphere and they all contain its centre. More generally, a chord of a convex body in $\mathbf{R}^{d}$ is called a diameter if there are two parallel supporting hyperplanes at the two endpoints of the chord.

It is easily seen that there are points on at least two diameters. From a result of Kosiński [6] proved in a more general setting it follows that every convex body has a point lying on at least three diameters. Does a typical convex body behave like a sphere and contain a point on infinitely or even uncountably many diameters?

But what is a typical convex body? The space $\mathcal{K}$ of all convex bodies $(d$ dimensional compact convex sets) in $\mathbf{R}^{d}$, equipped with the Hausdorff metric, is a Baire space. Its subspace $\mathcal{K}^{1}$ of all convex bodies which are smooth and strictly convex is residual (see [5]) and is therefore itself a Baire space. We say that typical elements or most elements of a Baire space enjoy some property if all those enjoying it form a residual set.

It is known that in most planar convex bodies most points lie on infinitely many diameters ([9]). This is in striking contrast with the fact that in the measuretheoretical sense almost every point in any planar convex body lies on finitely many diameters [3] and with the fact that in many usual planar convex bodies every point lies on at most three diameters. Unfortunately, it does not seem that the method of proof used in [9] can be generalized to higher dimensions.

In $\mathbf{R}^{d}$, using other methods we prove that in most convex bodies most points lie on infinitely many diameters. Moreover, we show that in most convex bodies infinitely many points lie on uncountably many diameters. To a question of Hammer [2] concerning points on uncountably many diameters an answer was provided long ago by Besicovitch and Zamfirescu [1]. This answer will now be strengthened and put into a new light.

For a survey of results on typical convex bodies, see [10]. We use the following notations: $x y$ means the line segment from $x$ to $y . N_{K}(x)$, or simply $N(x)$, is the set of outer unit normals of $K$ at $x \in \operatorname{bd} K$, where bd $K$ is the boundary of $K$. For $K \in \mathcal{K}^{1}, N(x)$ consists of a single point which we denote by $a(x)$. The interior of $K$ is denoted by int $K$.

For $M \subset \mathbf{R}^{d}, \operatorname{diam} M=\sup \{\|x-y\|: x, y \in M\}$.

A lemma. Let $0<\lambda<1$ and consider a diameter $x y$ of $K \in \mathcal{K}$. Set

Received August 9, 1988. 
$z=\lambda x+(1-\lambda) y$ and

$$
\begin{aligned}
& K_{\lambda}=z+\frac{1-\lambda}{\lambda}(z-K), \\
& R_{1}=K \backslash \text { int } K_{\lambda}, \\
& R_{2}=K_{\lambda} \backslash \text { int } K .
\end{aligned}
$$

Actually $R_{1}$ and $R_{2}$ depend on $x, y$ and $\lambda$, but we will suppress this dependence in the notation. Notice that $x \in R_{1}$ and $x \in R_{2}$. We call a diameter $\lambda$-regular if neither $R_{1}$ nor $R_{2}$ includes a continuum containing $x$ and different $\{x\}$. A diameter is regular if it is $\lambda$-regular for all $\lambda \in(0,1)$.

It is easy to see that no diameter of the unit ball in $\mathbf{R}^{d}$ is regular. We give an example of a $\lambda$-regular diameter with $\lambda=1 / 2$ as follows. Define a function $f:[-1,1] \rightarrow R$ by

$$
f(x)= \begin{cases}\sqrt{1-x^{2}} & \text { if } x \in\left[-1, \frac{1}{2}\right] \cup\left\{\frac{3}{4}, \frac{7}{8}, \frac{15}{16}, \ldots\right\} \cup\{1\} \\ \min _{i} g_{i}(x) & \text { otherwise, }\end{cases}
$$

where $g_{i}$ is the linear function that coincides with $f$ at

$$
x=\left(2 \cdot 4^{i-1}-1\right) / 2 \cdot 4^{i-1} \text { and } x=\left(4^{i}-1\right) / 4^{i} \quad(i=1,2,3, \ldots) .
$$

Clearly, $f$ is concave. Now let $K \subset \mathbf{R}^{d}$ be the convex body obtained by rotating the graph of $f$ around the $x$-axis. It follows from this construction that the interval $[-1,1]$ on the $x$-axis is a $1 / 2$-regular diameter of $K$.

Lemma. For most convex bodies $K \in \mathcal{K}$ and for most points $x \in \operatorname{bd} K$ there is a unique diameter $x y$ and this diameter is regular.

Proof. We show uniqueness first, which is easy. $\mathcal{K}^{1}$ is residual in $\mathcal{K}$ (see [5]). Take $K \in \mathcal{K}^{1}$ and $x \in \operatorname{bd} K$. Then $a(x)$ is uniquely determined and there is a unique $y \in \mathrm{bd} K$ with $a(y)=-a(x)$. Thus $x y$ is a diameter and it is the only diameter that contains $x$.

Let $\alpha \in(0,1 / 2), \beta>0$ and define, for $K \in \mathcal{K}^{1}$,

$$
\begin{aligned}
S_{\alpha}(K)= & \{x \in \text { bd } K: \text { the diameter } x y \text { is not } \\
& \lambda-\text { regular for some } \lambda \in[\alpha, 1-\alpha]\}, \\
S_{\alpha, \beta}(K)= & \left\{x \in S_{\alpha}(K): \exists \text { continuum } C \ni x \text { in } R_{1} \text { or } R_{2}\right. \text { with } \\
& \operatorname{diam} C \geqq \beta \text { for some } \lambda \in[\alpha, 1-\alpha]\} .
\end{aligned}
$$

A routine argument shows that $S_{\alpha, \beta}(K)$ is closed. Clearly

$$
S(K)=\bigcup_{\alpha>0} \bigcup_{\beta>0} S_{\alpha, \beta}(K)
$$


is in the set of endpoints of non-regular diameters. Set

$$
\mathcal{K}^{0}=\left\{K \in \mathcal{K}^{1}: S(K) \text { is of second category }\right\} \text {. }
$$

Choosing sequences $\alpha_{i} \rightarrow 0$ and $\beta_{j} \rightarrow 0, S_{\alpha_{i}, \beta_{j}}(K)$ cannot all be nowhere dense if $K \in \mathcal{K}^{0}$. Then, for some $i$ and $j, S_{\alpha_{i}, \beta_{j}}(K)$ must contain a "disk"

$$
D=\operatorname{bd} K \bigcap B(x, \gamma)
$$

where $x \in \operatorname{bd} K, \gamma>0$ and $B(x, \gamma)$ stands for the ball with radius $\gamma$ and centre $x$. Set

$$
\mathcal{K}_{\alpha, \beta, \gamma}=\left\{K \in \mathcal{K}^{1}: S_{\alpha, \beta}(K) \text { contains a disk of radius } \gamma\right\} \text {. }
$$

Clearly, for some sequence $\gamma_{k} \rightarrow 0$,

$$
\mathcal{K}^{0}=\bigcup_{i} \bigcup_{j} \mathcal{K}_{\alpha_{i}, \beta_{j}, \gamma_{k}}
$$

Now we show that $\mathcal{K}_{\alpha, \beta \gamma}$ is nowhere dense in $\mathcal{K}$. This will prove the lemma. We (again) omit the proof of the following claim: $\mathcal{K}_{\alpha, \beta, \gamma}$ is closed.

Now let $O$ be an open set in $\mathcal{K} \backslash \mathcal{K}_{\alpha, \beta, \gamma}$. Take a smooth and strictly convex body $K \in O$. Choose a finite set $X \subset$ bd $K$ with the property that any disk $D \subset$ bd $K$ of radius $\gamma / 2$ contains a point of $X$. This is clearly possible. For each $x$, let $x y$ be the unique diameter through $x$. Cut off a small cap $C_{x}$ (and $C_{y}$ ) from $K$ by a hyperplane orthogonal to $a(x)$ and close to $x$ (y, respectively). These cuts can be chosen so small that the caps $C_{x}, C_{y}(x \in X)$ are all pairwise disjoint, and the diameter of each $C_{x}$ and $C_{y}$ is less than $\gamma / 2$. Moreover, if the cuts are small enough, then

$$
K^{\prime}=K \backslash \bigcup_{x \in X}\left(C_{x} \cup C_{y}\right) \in O
$$

and any $K^{*} \in \mathcal{K}$ with $\mathcal{K}^{\prime} \subset K^{*} \subset K$ belongs to $O$, too. The diameter $x y$ intersects bd $K^{\prime}$ in points $u$ and $v$ where $u \in C_{x}$ and $v \in C_{y}$. Let $B(u)[B(v)]$ be a $(d-1)$-dimensional ball with centre $u[v]$ and radius $\rho$, lying in the bounding hyperplane of $C_{x}\left[C_{y}\right]$. The radius $\rho$ can be chosen so small that $B(u) \subset C_{x}$ and $B(v) \subset C_{y}$ for all $x \in X$.

Define two functions $f, g:[0, \infty) \rightarrow[0, \infty)$ by

$$
\begin{aligned}
& f(t)= \begin{cases}t^{4} & \text { if } t \leqq \epsilon, \\
t^{2}-\epsilon^{2}+\epsilon^{4} & \text { if } t>\epsilon,\end{cases} \\
& g(t)=t^{3},
\end{aligned}
$$

where $\epsilon$ is to be specified later. 
Let $z \in \mathbf{R}^{d}$ and write

$$
z-u=z^{+}+\zeta a
$$

where $a=a(x)$ and $z^{+}$is the component of $z-u$ orthogonal to $a$. Define the set

$$
F_{x}=\left\{z \in \mathbf{R}^{d}:\left\|z^{+}\right\| \leqq \rho, 0 \leqq \zeta \leqq \nu-\mu f\left(\left\|z^{+}\right\|\right)\right\},
$$

where $\mu>0$ and $\nu>0$ will be chosen so small that, firstly, $F_{x} \subset C_{x}$ and, secondly, no hyperplane touching $F_{x}$ at some point $z$ with $\left\|z^{+}\right\|<\rho, \zeta=$ $\nu-\mu f\left(\left\|z^{+}\right\|\right.$) meets $K \backslash C_{x}$ (for all $x \in X$ ). Similarly, let $w \in R^{d}$ and set

$$
w-v=w^{+}+\omega b
$$

where $b=-a$ is the outer normal to $K$ at $y$ and $w^{+}$is the component of $w-v$ orthogonal to $b$. Define

$$
G_{y}=\left\{w \in R^{d}:\left\|w^{+}\right\| \leqq \rho, 0 \leqq \omega \leqq \nu-\mu g\left(\left\|w^{+}\right\|\right)\right\},
$$

where, again, $\nu>0$ and $\mu>0$ are chosen so that $G_{y} \subset C_{y}$ and no hyperplane, touching $G_{y}$ at some point $w$ with $\left\|w^{+}\right\|<\rho, \omega=\nu-\mu g\left(\left\|w^{+}\right\|\right)$meets $K \backslash C_{y}$. Clearly, $\nu$ and $\mu$ can be chosen the same for all $F_{x}$ and $G_{y}$.

Define now

$$
K^{*}=\operatorname{conv}\left(K^{\prime} \cup \bigcup_{x \in X}\left(F_{x} \cup G_{y}\right)\right)
$$

Evidently $K \supset K^{*} \supset K^{\prime}$ and so $K^{*} \in O$. We claim that $K^{*} \notin \mathcal{K}_{\alpha, \beta, \gamma}$.

Consider $x \in X$ and the points $y, u, v$ defined above. Set

$$
x^{*}=u+\nu a, \quad y^{*}=v+\nu b .
$$

By the construction, $x^{*} y^{*}$ is a diameter of $K^{*}$ with outer normals $a$ and $b$ at its endpoints. We will now show that for $x^{*}=\lambda x^{*}+(1-\lambda) y^{*}$ with $\alpha<\lambda<1-\alpha$ neither $R_{1}^{*}=K^{*} \backslash$ int $K_{\lambda}^{*}$ nor $R_{2}^{*}=K_{\lambda}^{*} \backslash$ int $K^{*}$ contains a continuum of diameter $\beta$ containing $x^{*}$.

By construction, bd $K^{*}$ coincides with a piece of bd $F_{x}\left[\mathrm{bd} F_{y}\right]$ in a neighbourhood of radius $\rho$ of $x^{*}\left[y^{*}\right]$. Then bd $K$ is rotationally symmetric in a small neighbourhood of $x^{*}\left[y^{*}\right]$, the axis of symmetry being the line though $x^{*}\left[y^{*}\right]$ with direction $a$. Thus $R_{1}^{*}$ and $R_{2}^{*}$ are also rotationally symmetric.

It is easy to reduce our question now to a question about the functions $f$ and $g: R_{1}^{*}$ and $R_{2}^{*}$ contain a continuum of diameter at least $\beta$ containing $x^{*}$ if and only if either

$$
f(t) \geqq \frac{\lambda}{1-\lambda} g\left(\frac{1-\lambda}{\lambda} t\right)
$$


on the interval $[0, \beta / 2]$, or

$$
f(t) \leqq \frac{\lambda}{1-\lambda} g\left(\frac{1-\lambda}{\lambda} t\right)
$$

on the same interval. We show now that neither of these two possibilities holds. Write $\sigma=(1-\lambda) / \lambda$; then

$$
\alpha /(1-\alpha)<\sigma<(1-\alpha) / \alpha
$$

Clearly

$$
f(t)=t^{4}<\frac{1}{\sigma} g(\sigma t)=\gamma^{2} t^{3}
$$

if $0 \leqq t<\sigma^{2} /(1-\alpha)^{2}$, and we choose $0<\epsilon<\alpha^{2} /(1-\alpha)^{2}$. Now for $t=2 \epsilon$

$$
\begin{aligned}
f(t) & =3 \epsilon^{2}+16 \epsilon^{4} \\
\frac{1}{\sigma} g(\sigma t) & =\sigma^{2} \cdot 8 \epsilon^{3} \leqq\left(\frac{1-\alpha}{\alpha}\right) \cdot 8 \epsilon^{3},
\end{aligned}
$$

and so

$$
\frac{1}{\sigma} g(\sigma t)<f(t) \text { if } \epsilon<\frac{3}{8}\left(\frac{\alpha}{1-\alpha}\right)^{2}
$$

We also have to have $t=2 \epsilon<\beta / 2, t=2 \epsilon<\rho$ and $\gamma t=\gamma^{2} \epsilon<\rho$. Thus, choosing

$$
\epsilon=\frac{1}{2} \min \left\{\frac{3}{8}\left(\frac{\alpha}{1-\alpha}\right)^{2}, \beta / 4, \rho / 2 \frac{\rho \alpha}{2(1-\alpha)}\right\}
$$

there will be no continuum, of diameter at least $\beta$ and containing $x^{*}$ in either of the sets $R_{1}^{*}$ and $R_{2}^{*}$ for all $x \in X$.

Assume now that there is a disk $D^{*} \subset S_{\alpha, \beta}\left(K^{*}\right)$ of radius $\Gamma$, with centre $z^{*} \in K^{*}, z^{*} \in C_{x}$ (or $C_{y}$ ) would imply $x^{*} \in D^{*}$ (or $y^{*} \in D^{*}$ ) because $C_{x}$ and $C_{y}$ have diameter less then $\beta / 2$. But $x^{*} \in D^{*} \subset S_{\alpha, \beta}\left(K^{*}\right)$ contradicts what we just established. So $x z^{*} \in$ bd $K$, and, by the choice of $X,\left\|z^{*}-x\right\|<\gamma / 2$ for some $x \in X$. The diameter of $C_{x}$ is less than $\gamma / 2$, so $\left\|x-x^{*}\right\|<\gamma / 2$ whence

$$
\left\|z^{*}-x^{*}\right\| \leqq\left\|z^{*}-x\right\|+\left\|x-x^{*}\right\|<\gamma
$$

and $x^{*} \in D^{*} \subset S_{\alpha, \beta}\left(k^{*}\right)$, a contradiction. 


\section{Points on infinitely many diameters.}

THEOREM 1. In most convex bodies, on most diameters, each point belongs to infinitely many diameters.

Proof. Let $K$ be a smooth convex body with the properties of the lemma. Also, let $M_{\alpha}$ be the set of points in $\mathbf{R}^{d}$ lying on a least $\alpha$ diameters of $K$.

Consider a regular diameter $x y$ of $K$. For $\lambda \in(0,1)$, let

$$
\begin{aligned}
z & =\lambda x+(1-\lambda) y, \\
K_{\lambda} & =z+\frac{1-\lambda}{\lambda}(z-K) .
\end{aligned}
$$

For $v \in$ bd $K$ close to $x$, let $v w$ be a diameter of $K$ and

$$
v^{\prime}=z+\frac{1-\lambda}{\lambda}(z-w) \text {. }
$$

Put

$$
f_{z}(v)=\|v-z\|-\left\|v^{\prime}-z\right\|
$$

From now on we assume $d \geqq 3$; the case $d=2$ can be dealt with analogously (or see [9]). By the definition of a regular diameter, in any neighbourhood of $x$ there is a component $V^{+}$of $f_{z}^{-1}\left(\mathbf{R}^{+}\right)$and a component $V^{-}$of $f_{z}^{-1}\left(\mathbf{R}^{-}\right)$both surrounding $x$, i.e., such that $x$ lies in a bounded component $V^{\prime+}$ of $C V^{+}$and in a bounded component $V^{\prime-}$ of $C V^{-}$. Now $\rho_{z}$ has in every component $V^{+}$a local maximum. Since every local maximum is an endpoint of a diameter through $z, z \in M_{\aleph_{0}}$. By the Lemma, the set of regular diameters is residual. Thus the theorem is proved.

THEOREM 2. In most convex bodies, most points belong to infinitely many diameters.

Proof. As a consequence of the proof of Theorem 1, we find $k$ components $V_{1}^{+}, \ldots, V_{k}^{+}$of $f_{z}^{-1}\left(\mathbf{R}^{+}\right)$and $k-1$ components $V_{1}^{-}, \ldots, V_{k-1}^{-}$of $f_{z}^{-1}\left(\mathbf{R}^{-}\right)$such that $V_{i}^{-} \subset V_{i}^{\prime+}$ and $V_{i+1}^{+} \subset V_{i}^{\prime-} \quad(i=1, \ldots, k-1)$. Since $v^{\prime}$ depends continuously on $v$ and $v$ and $z$, so does $f_{z}(v)$, which implies that for some neighbourhood $N_{i}$ of $z$ we have, for any $u \in N_{i}$, two components $U_{i}^{+}, U_{i+1}^{+}$of $f_{u}^{-1}\left(\mathbf{R}^{+}\right)$and a component $U_{i}^{-}$of $f_{u}^{-1}\left(\mathbf{R}^{-}\right)$such that $U_{i}^{-}$lies in a bounded component $U_{i}^{\prime+}$ of $C U_{i}^{+}$and $U_{i+1}^{+}$lies in a bounded component $U_{i}^{\prime-}$ of $C U_{i}^{-}$. Thus, for any $u \in U_{i=1}^{k} N_{i}, U_{i}^{-} \subset U_{i}^{\prime+}$ and $U_{i+1}^{+} \subset U_{i}^{\prime-} \quad(i=1, \ldots, k-1)$. It follows that, for any such $u, f_{u}(v)$ has at least $k$ local maxima (in the variable $v$ ). Every such local maximum is the endpoint of a diameter through $u$. This means that

$$
\bigcap_{i=1}^{k} N_{i} \subset M_{k} .
$$


Now let $O$ be open in $K$. Since every point of $K$ lies on a diameter, there is a diameter meeting $O$. By Theorem 1 , there also is a regular diameter $x y$ with a point $z \in x y \cap O$. Now, the considerations above imply that $C M_{k}$ is nowhere dense, since

$$
O \cap \bigcap_{i=1}^{k} N_{i} \subset M_{k} .
$$

Thus

$$
C M_{\aleph_{0}}=\mathcal{C} \bigcap_{k=1}^{\infty} M_{k}=\bigcup_{k=1}^{\infty} C M_{k}
$$

is of first category and the theorem is proved.

Points on uncountably many diameters. Theorems 1 and 2 leave open the question whether a typical convex body must have a point lying on uncountably many diameters.

For any $K \in \mathcal{K}$ and $z \in K$, let $\Omega(z)$ be the set of all unit vectors $u \in S^{d-1}$ such that the chord through $z$ in direction $u$ is a diameter of $K$. Clearly, $\Omega(z)=-\Omega(z)$.

THEOREM 3. Let $S \subset \mathbf{R}^{d}$ be countable. For most convex bodies $K$, at each point $z \in S \cap K, \Omega(z)$ is perfect and nowhere dense.

Let $z \in S$. We prove the theorem for $S=\{z\}$; since any countable intersection of residual sets is residual, the theorem in its general form will follow. We start by observing that $z \in$ bd $K$ for a nowhere dense family of sets $K$ only. So we may assume $z \in$ int $K$. We prove that, for most $K \in \mathcal{K}$, the set $\Omega(z)$ is perfect and nowhere dense. First we remark that $\Omega(z)$ is always closed. Thus we only have to prove that $\Omega(z)$ contains no open set and no isolated element, for most $K$. In fact the nowhere density follows from Theorem 5. However we give here a simple direct proof.

Suppose $\Omega(z)$ includes an open set. This means that there are two diametrically opposite points $x, y$ with $z \in x y$ and two open sets $F_{x}, F_{y}$ around $x$ and $y$ on bd $K$ such that $F_{x}$ and $F_{y}$ are inversely homothetical with respect to $z$. Let

$$
\mathcal{K}_{n}=\left\{K \in \mathcal{K}: \exists F_{x}, F_{y} \text { such that } \operatorname{diam} F_{x} \geqq n^{-1}, \operatorname{diam} F_{y} \geqq n^{-1}\right\} .
$$

Clearly, $\mathcal{K}_{n}$ is closed; it remains to show that $\mathcal{C} \mathcal{K}_{n}^{\prime}$ is dense. This is indeed so since any convex body is approximable by a polytope without parallel facets.

Suppose now $\Omega(z)$ has an isolated element $\omega$. Then there is a disk $G_{x} \subset$ bd $K$ around an endpoint $x$ of the diameter $x y$ through $z$ in direction $\omega$ such that the only diameter through $z$ with an endpoint in $G_{x}$ is $x y$. Let

$$
\mathcal{K}_{n}=\left\{K \in \mathcal{K}: \exists G_{x} \text { such that diam } G_{x}>n^{-1}\right\} .
$$

We show that $\mathcal{K}_{n}$ is nowhere dense. Let $O \subset \mathcal{K}$ be open. 
Take $K^{\prime} \in O$ to be smooth. $\Omega(z)$ is compact, so we may choose a finite open covering of $\Omega(z)$ with sets $D_{1}, \ldots, D_{m}$ of diameter less than $(4 n)^{-1}$. Consider a point

$$
\omega \in S^{d-1} \backslash \bigcup_{i=1}^{m} D_{i} .
$$

Since $\omega \notin \Omega(z)$, the chord $x_{\omega} y_{\omega}$ in direction $\omega$ satisfies

$$
a\left(x_{\omega}\right)+a\left(y_{\omega}\right) \neq 0
$$

and in a small neighbourhood $D(\omega)$ of $\omega$ we have

$$
\left\|a\left(x_{v}\right)+a\left(y_{v}\right)\right\|>\eta(\omega)
$$

for some $\eta(\omega)>0$ and all $v \in D(\omega)$. Choose a finite covering $D\left(\omega_{1}\right), \ldots, D\left(\omega_{l}\right)$ of $S^{d-1} \backslash \bigcup_{i=1}^{m} D_{i}$ and let

$$
\eta=\min \left\{\eta\left(\omega_{i}\right): i=1, \ldots, l\right\}
$$

Now choose a polytope $P$ very close to $K^{\prime}$, so that

$$
\left\|v^{\prime}+v^{\prime \prime}\right\| \geqq \eta / 2
$$

for any $v^{\prime} \in N_{p}\left(x_{v}\right), v^{\prime \prime} \in N_{p}\left(y_{v}\right)$, and any

$$
v \in \bigcup_{i=1}^{l} D\left(\omega_{i}\right)
$$

and so that $P$ has a diameter $x_{i} y_{i}$ in a direction $u_{i} \in D_{i}$ for any $i=1, \ldots, m$. These diameters can be chosen so that $x_{i}$ is a vertex of $P$ and $y_{i}$ is in the relative interior of a facet $F_{i}$ of $P$. Now fix $i$ and denote $x_{i} y_{i}$ and $F_{i}$ by $x y$ and $F$ respectively (for simplicity of notation). Choose a line segment $x^{1} x^{2}$ parallel to $F$, so that $x$ is the midpoint of $x^{1} x^{2}$. Let $y^{1}\left[y^{2}\right]$ be the point in bd $P$ close to $y$ and collinear with $x^{1}$ and $z\left[x^{2}\right.$ and $\left.z\right]$.

We choose the line segment $x^{1} x^{2}$ so short that $y^{1}, y^{2} \in F$ and that $P^{\prime}=$ $\operatorname{conv}\left(P \cup\left\{x^{1}, x^{2}\right\}\right)$ is as close as we wish to $P$.

Now we take $n^{1} \in$ int $N_{P^{\prime}}\left(x^{1}\right)$ and $n^{2} \in$ int $N_{P^{\prime}}\left(x^{2}\right)$ very close to $-a(y)$ and we cut off from $P^{\prime}$ two caps by hyperplanes through $y$ orthogonal to $n^{1}$, respectively $n^{2}$. We get in this way a convex polytope $Q$ as close to $P^{\prime}$ as we wish.

Denote by $\bar{y}^{j}$ the point in bd $Q$ collinear with $x^{j}$ and $z \quad(j=1,2)$. It is clear that if the segment $x^{1} x^{2}$ is short enough then $x^{1} \bar{y}^{1}$ and $x^{2} \bar{y}^{2}$ will be diameters of $Q$.

Let $H_{x}, H_{y}$ be the hyperplanes through $x$, respectively $y$, both parallel to $F$. Now define a function $f_{Q}$ on $H_{x}$ : for $t \in H_{x}$, let $x(t) \in$ bd $Q$ be the point close 
to $x$ with $x(t)-t$ orthogonal to $H_{x}$ (or zero) then determine $y(t) \in$ bd $Q$ collinear with $z$ and $x(t)$. Next determine $s(t) \in H_{y}$ as the orthogonal projection of $y(t)$ to $H_{y}$. Finally let

$$
f_{Q}(t)=\|x(t)-t\|-\frac{\|x(t)-z\|}{\|y(t)-z\|}\|y(t)-s(t)\| .
$$

By the construction, this function $f_{Q}$ has strict local minima at $x^{1}$ and $x^{2}$. Now we do this same thing successively with all diameters $x_{i} y_{i}(i=1, \ldots, m)$, not disturbing the properties of the polytopes and functions already constructed, which are of interest for our purposes.

We eventually get a polytope $P^{*} \in O$. We claim that, if $K$ is in a neighbourhood $\mathcal{N}$ of $P^{*}$ which is small enough, then for every diameter of $K$ through $z$ there will be another one with angular distance less than $n^{-1}$. If $K$ is close enough to $P^{*}$ then $K$ cannot have a diameter through $z$ in any direction from $\bigcup_{i=1}^{1} D\left(\omega_{i}\right)$. So every diameter of $K$ through $z$ is defined by a direction from $D_{i}$, for some $i \in\{1, \ldots, m\}$. We show that there are two directions of diameters through $z$ in every $D_{i}$ and this will prove the claim. Consider the function $f_{K}$ defined in the same way as before, but with $K$ instead of $Q$.

If $K$ is close enough to $P^{*}$, then $f_{K}$ will be very close to $f_{P^{*}}$ and so $f_{K}$ will have at least two local minima $t_{1}$ and $t_{2}$, close to $x^{1}$ and $x^{2}$. At these local minima, the corresponding chords $x_{K}\left(t_{j}\right) y_{K}\left(t_{j}\right)(j=1,2)$ will be diameters of $K$. Thus

$$
O \cap \mathcal{N} \cap \mathcal{K}_{n}=\emptyset
$$

and the proof is finished.

In 1965 Hammer [2] raised the question whether there exist a convex body $K$ and a point $z \in$ int $K$ such that the set $R(z)$ of all ratios into which $z$ divides the various diameters through $z$ is uncountable. Besicovitch and Zamfirescu [1] answered the question by providing such a convex body and such an interior point.

THEOREM 4. Let $S \subset \mathbf{R}^{d}$ be countable. For most convex bodies $K$, at each point $z \in S \cap K, R(z)$ is uncountable.

Proof. Let $z \in S$. We prove the theorem for $S=\{z\}$. First we remark that $\{K \in \mathcal{K}: z \in \mathrm{bd} K\}$ is nowhere dense. We prove that, for most $K \in \mathcal{K}$ with $z \in \operatorname{int} K, R(z)$ is perfect. Clearly, $R(z)$ is closed. Thus we only have to show that $R(z)$ contains no isolated point, for most $K \in \mathcal{K}$. The proof parallels the last part of the proof of Theorem 3, where it is shown that, typically, $\Omega(z)$ has no isolated point.

The only change which has to be made is the following: Using the notations of the mentioned proof, we have to arrange, when choosing the unit vectors $n^{1}$ and $n^{2}$ in int $N_{P^{\prime}}\left(x^{1}\right)$ and int $N_{P^{\prime}}\left(x^{2}\right)$ respectively, that

$$
\frac{\left\|x^{1}-z\right\|}{\left\|\bar{y}^{1}-z\right\|} \neq \frac{\left\|x^{2}-z\right\|}{\left\|\bar{y}^{2}-z\right\|} \text {. }
$$


Directions of concurrent diameters. In Theorem 3 it is shown that, for countably many points $z$, the set $\Omega(z)$ is nowhere dense. This can be considerably improved by allowing $z$ to be any point in $K$ and by replacing nowhere density by uniform porosity.

A set $M$ in a metric space $(X, \delta)$ is called uniformly porous if there exists a constant $\alpha>0$ such that in any ball $B(x, r) \subset X$ there is a point $y$ such that

$$
B(y, \alpha \delta(x, y)) \cap M=\emptyset .
$$

THEOREM 5. For most convex bodies $K \in \mathcal{K}$ and for all points $z \in K, \Omega(z)$ is uniformly porous.

Proof. Suppose $d \geqq 3$. The proof for $d=2$ is analogous and simpler. Define $K(\epsilon)$ to be the inner parallel body to $K$ at distance $\epsilon$. We denote the angle between the vectors $u, v \in S^{d-1}$ by $\Varangle u v$. Let $i(S)$ and $\mu(S)$ be the inradius and the $(d-2)$-dimensional measure of the convex set $S \subset S^{d-2}$ (the convexity is understood on $S^{d-2}$ ). Since $i(S) \rightarrow 0$ implies $\mu(S) \rightarrow 0$, the number

$$
\alpha=\inf \left\{i(S): \mu(S)=d^{-2} \mu\left(S^{d-2}\right)\right\}
$$

is positive. Set now

$$
\begin{gathered}
\mathcal{K}_{n}=\left\{K \in \mathcal{K}: \exists z \in K\left(n^{-1}\right) \text { and } \exists u \in S^{d-1} \text { such that } \forall v \in S^{d-1}\right. \\
\text { with } \left.\Varangle u v \leqq n^{-1}, \exists w \in \Omega(z) \text { with } \Varangle w v \leqq \frac{\alpha}{8} \cdot \Varangle u v\right\} .
\end{gathered}
$$

Obviously, $\mathcal{K}_{n}$ is closed.

We show now that $\mathcal{K}_{n}$ is nowhere dense. Let $O \subset \mathcal{K}$ be an open set. If there is no $K \in O$ with $K\left(n^{-1}\right) \neq \emptyset$ then $O \cap \mathcal{K}_{n}=\emptyset$ and we are done. If this is not the case, then there is a simple polytope $P \in O$ without parallel facets or facets of diameter at least $n^{-1}$, and such that $P\left(n^{-1}\right) \neq \emptyset$. Consider now $z \in P\left(n^{-1}\right)$ and a chord $x y$ of $P$ through $z$ with direction $u$. If $x y$ is not a diameter of $P$ then the chords through $z$ that are close enough to $x y$ are not diameters either.

So assume $x y$ is a diameter. Then $x$ or $y$ or both are contained in faces of $P$, each of dimension less than $d-1$, for $P$ has no parallel facets. Let $F_{1}, \ldots, F_{k}$ and $G_{1}, \ldots, G_{l}$ be the facets containing $x$ and $y$ respectively. Let $H$ be the hyperplane through $x$ with normal $u$. Project $F_{1}, \ldots, F_{k}$ from $z$ to $H$, then reflect $G_{1}, \ldots, G_{l}$ through $z$ and project the resulting sets from $z$ to $H$. In this way we get $(d-1)$ dimensional polytopes $F_{1}^{\prime}, \ldots, F_{k}^{\prime}$ and $G_{1}^{\prime}, \ldots, G_{l}^{\prime}$ in $H$ (because the diameter of each facet is less than $n^{-1}$ ). Let

$$
F_{i}^{\prime \prime}=\left\{\left\|x^{\prime}-x\right\|^{-1}\left(x^{\prime}-x\right): x^{\prime} \in F_{1}^{\prime}\right\} \quad(i=1, \ldots, k)
$$


and define $G_{j}^{\prime \prime} \quad(j=1, \ldots, l)$ analogously. These sets are convex in $S^{d-2}$. Clearly, the convex sets $F_{i}^{\prime \prime} \cap G_{j}^{\prime \prime}(i=1, \ldots, k, j=1, \ldots, l)$ form a subdivision of $S^{d-2}$. Now $P$ is simple, so $k \leqq d$ and $l \leqq d$. Thus there are $i$ and $j$ so that

$$
\mu\left(F_{i}^{\prime \prime} \cap G_{j}^{\prime \prime}\right) \geqq d^{-2} \mu\left(S^{d-2}\right) .
$$

Then $F_{i}^{\prime \prime} \cap G_{j}^{\prime \prime}$ contains a $(d-2)$-dimensional (spherical) disk of radius $\alpha$ and centre $c$. Consider the point $c_{\lambda}=x+\lambda c$. For small enough $\lambda>0$, the open $(d-1)$-dimensional ball $D$ in $H$ around $c_{\lambda}$ of radius $\alpha\left\|c_{\lambda}-x\right\| / 2$ lies in $F_{i}^{\prime} \cap G_{j}^{\prime}$. Choose

$$
v=\left\|c_{\lambda}-z\right\|^{-1}\left(c_{\lambda}-z\right)
$$

We claim that, for any $w \in S^{d-1}$ with $\Varangle v w \leqq(\alpha / 8) \Varangle u v$, the halfline $\{z+\lambda w$ : $\lambda \geqq 0\}$ intersects $H$ inside $D$.

Indeed, let $S^{*}$ be the sphere with centre $z$ which passes through $x$ and let $v^{\prime}$ be the intersection of $z c_{\lambda}$ with $S^{*}$. Let $w^{\prime}$ and $w^{\prime \prime}$ be the intersections of the ray from $z$ in direction $w$ with $S^{*}$ and $H$, respectively. Let $w_{0}$ be the point of $z w^{\prime \prime}$ nearest to $v^{\prime}$. Then

$$
\frac{\left\|v^{\prime}-w_{0}\right\|}{\left\|c_{\lambda}-x\right\|}=\cos \Varangle u w \cdot \cos \Varangle u v \geqq \cos n^{-1} \cdot \cos (2 n)^{-1}>\cos ^{2} 1>\frac{1}{4} .
$$

But

$$
\frac{\left\|v^{\prime}-w_{o}\right\|}{\left\|c_{\lambda}-x\right\|} \leqq \frac{v^{\prime} w^{\prime}}{v^{\prime} x} \leqq \frac{\Varangle v w}{\Varangle u v} \leqq \frac{\alpha}{8}
$$

Thus

$$
\left\|c_{\lambda}-w^{\prime \prime}\right\|<4\left\|v^{\prime}-w_{0}\right\| \leqq \frac{\alpha}{2}\left\|c_{\lambda}-x\right\|
$$

which verifies our claim.

Now, $D \subset F_{i}^{\prime} \cap G_{j}^{\prime}$. Since $F_{i}$ and $G_{j}$ are not parallel, $w \notin \Omega(z)$. Hence $P \subseteq \mathcal{K}_{n}$ and $\mathcal{K}_{n}$ is nowhere dense.

It follows that

$$
\mathcal{K}^{1} \cap\left(\mathcal{K} \backslash \bigcup_{n=1}^{\infty} \mathcal{K}_{n}\right)
$$

is residual. Let $K$ be an element of this set and take $z \in K$. If $z \in$ int $K$, then $z \in K\left(n^{-1}\right)$ for some $n$ and $\Omega(z)$ is uniformly porous. If $z \in \operatorname{bd} K$, then $\Omega(z)$ consists of two (antipodal) points only. The theorem is proved. 
Remark. It follows from Theorem 5 and from Lebesgue's density theorem that, for most $K \in \mathcal{K}$ and any $z \in K, \Omega(z)$ has measure zero on $S^{d-1}$.

Normals to typical convex surfaces. We shall now consider normal lines to convex surfaces. Heil [4] proved that any convex surface in $\mathbf{R}^{d}$ admits a point lying on at least 6 normals to the surface for any dimension $d \geqq 3$. For a typical convex surface and any $d \geqq 2$ the following holds.

TheOREM. ([7], [8]) For most convex surfaces, most points in $\mathbf{R}^{d}$ lie on infinitely many normals.

We are now able to complete the generic description of normals to convex surfaces.

Let $\Phi(z)$ denote the set of directions of normals through the point $z \in \mathbf{R}^{d}$ to a given convex surface.

Theorem 6. Let $S \subset \mathbf{R}^{d}$ be countable. For most convex surfaces in $\mathbf{R}^{d}$ the following is true:

(i) For any point $z \in S, \Phi(z)$ is perfect in $S^{d-1}$.

(ii) For any point $z \in \mathbf{R}^{d}, \Phi(z)$ is uniformly porous in $S^{d-1}$.

Theorem 6 admits a proof which is very similar and slightly simpler than those of Theorems 3 and 5 and will therefore be omitted.

Acknowledgement. Thanks are due to the referee for his (her) most valuable and detailed criticism.

\section{REFERENCES}

1. A. S. Besicovitch and T. Zamfirescu, On pencils of diameters in convex bodies, Rev. Roum. Math. Pures Appl. 11 (1966), 637-639.

2. P. C. Hammer, Problem 14 in colloquium on Convexity, Copenhagen (1965).

3. P. C. Hammer and A. Sobczyk, Planar line families II, Proc. Amer. Math. Soc. 4 (1953), 341-349.

4. E. Heil, Concurrent normals and critical points under weak smoothness assumptions, Ann. New York Acad. Sci. 440 (1985), 170-178.

5. V. Klee, Some new results on smoothness and rotundity in normed linear spaces, Math. Ann. 139 (1959), 51-63.

6. A Kosiński, On a problem of Steinhaus, Fund. Math. 46 (1958), 47-59.

7. T. Zamfirescu, Most convex mirrors are magic, Topology 21 (1982), 65-69.

8. Points on infinitely many normals to convex surfaces, J. Reine Angew. Math. 350 (1984), 183-187.

9. Intersecting diameters in convex bodies, Ann. Discrete Math. 20 (1984), 311-316.

10. Using Baire categories in geometry, Rend. Sem. Math. Univ. Politecn. Torino 43 (1985), 67-88.

Academy of Sciences,

Budapest, Hungary;

University of Dortmund,

Dortmund, Germany 